Rapid Reviews COVID-19

\title{
Reviews of "Genome sequencing of sewage detects regionally prevalent SARS-CoV-2 variants"
}

Pei-ying Hong ${ }^{1}$, Blake Wiedenheft ${ }^{2}$, Anna Nemudraia, Artem Nemudryy

${ }^{1}$ King Abdullah University of Science and Technology, Bese, Saudi Arabia,

${ }^{2}$ Montana State University Bozeman, Microbiology and Immunology

Published on: Jan 24, 2021

DOI: $10.1162 / 2$ e3983f5.328b02f9

License: Creative Commons Attribution 4.0 International License (CC-BY 4.0). 
Since our solicitation of reviews, this preprint has been published in mBio journal and the link to the published manuscript can be found here.

To read the original manuscript, click the link above.

Summary of Reviews: This preprint offers a successful demonstration of WGS-based detection of emerging SARS-CoV-2 variants in wastewater samples. Reviewers deemed major claims reliable, but experimental methodology and justification should be described in further detail.

\section{Reviewer 1 (Pei-ying Hong) |}

Reviewer 2 (Blake Wiedenheft, Anna Nemudraia, Artem Nemudryy) |



To read the reviews, click the links below. 